\title{
Modeling of the management of the microwave grain drying process
}

\author{
Sergey Morozov ${ }^{1}$, Konstantin Kuzmin ${ }^{1}$, Denis Pogodin $^{2, *}$, Lyudmila Kochetkova ${ }^{2}$ and Anna \\ Rogozhina $^{2}$ \\ ${ }^{1}$ Moscow state University of technology and management. K. G. Razumovsky (Smolensk branch), \\ Lenin street 77, Smolensk region, Vyazma, 215100, Russia \\ ${ }^{2}$ Moscow State University of Civil Engineering, 26, Yaroslavskoyeshosse, Moscow, 129337, Russia
}

\begin{abstract}
The article discusses the VisSim program, which allows simulating microwave grain drying process. Its capabilities and methods of development are shown, the structure of a mathematical model for management of the drying process and individual blocks is provided. It is noted that offered modeling of the grain drying process makes it possible to evaluate it as a technological process, as well as to show the possibility of its optimization. It should be noted that this method is environmentally friendly and less energy intensive than others.
\end{abstract}

\section{Introduction}

The task of finding the optimal modes of microwave installations operation is to find such laws of changes in control parameters that would ensure the best performance indicators of production activity - the highest quality of the finished product with the least expenditure of energy resources. To solve the problem of optimal control of the installation of milk pasteurization, knowledge of the basic laws governing their processes is necessary. Communication of these processes is reflected in the form of mathematical dependencies, called the mathematical model of the object.

Mathematical models reflecting the dynamics of an object's behavior are used to solve an optimal control problem.

The most productive method of constructing a mathematical model is experimentallyanalytical, when the model is based on defined mathematical dependencies (model structure), and then taking into account the experimental data, the model parameters are determined so that the calculated and experimental values differ minimally.

Since the production experiment is very expensive, research is carried out on laboratory installations. At the same time, an experiment using the classical principle of alternately change of each factor at a constant level of the others is ineffective. Before choosing a structure, it is necessary to identify the essential factors of their interaction, which determine the output state of the object.

\footnotetext{
*Corresponding author: PogodinDA@mgsu.ru
} 
Therefore, the goal of an experimental study of an object should obtain more information at a lower cost than it can be done by traditional classical methods. As is well known, this requirement is met by the conscious planning of the experiment, taking into account the conditions of the experiment.

It is quite natural that the task of determining the essential factors and their interactions can be solved in various ways.

Using models, it is possible to establish in each modeled process (object) the basic, inherent laws and to distract from auxiliary, secondary signs. When modeling, the simulated object is replaced by another ideal or material object, that ensured the profitability of the model experiment and the ability to transfer quantitative data from the model to the original. Models are divided into physical and mathematical.

Physical (material, instrument) models are process development units that are developed to measure the performance of various processes, polygons with the appropriate models for studying machines, models of devices, etc.

Physical modeling consists of conducting experimental studies on a model in which processes that are identical to the processes occurring in an object proceed. Then results of experiments transfer to a real object (original).

The VisSim program, developed by Visual Solutions, is an effective tool for modeling physical and technical objects, systems and their elements, which allows studying models of systems of a wide complexity range. VisSim is software for systems simulation, it has frequency, root, variation, neural tools for assessing quality, stability, synthesis, correction, optimization, linearization, programming of digital signal processors [1].

Models of systems and objects in the VisSim program are developed from separate blocks, virtual analogs of the physical element of the real system. VisSim's blocks can be divided into three main categories and one additional:

- $\quad$ blocks with only output: generators;

- $\quad$ blocks with both input and output: regenerator;

- $\quad$ blocks with only input: indicators;

- $\quad$ blocks without input and output.

An important component of the model is the connecting line - a virtual analogue of the physical connection of elements, transmitting effects from one element to another.

Development of a model from VisSim program blocks can be carried out by three methods:

1) according to the block schematic diagram of the studied system (differential equations should be represented in the form of transfer functions (TF));

2) according to the differential equations of the studied system relating to the required coordinate, usually relating to the error or the controlled variable;

3 ) according to the of first order differential equations describing the studied system under study (Cauchy form).

In addition, VisSim allows simulating discrete systems with multi-frequency quantization, i.e. one model may have discrete filters (blocks) with different sampling frequencies.

To develop models with multi-frequency quantization, it is sufficient to set the sampling periods not by default, but, if possible, many times larger. The simulation step must be an integer number of times less than any of the quantization periods $d T$ of discrete blocks.

Using the VisSim program it is possible to develop models consisting of both blocks of continuous and discrete time. Such systems are called hybrid. The signals in these models at the outputs of discrete blocks are modified only at the moment when the time quantum is completed, during which they remain unchanged. The signals at the outputs of continuous blocks are modified at each step. The time slice (sampling period) can be an integer number of times more than the simulation step. Like outputs, inputs of discrete blocks perceive a 
signal (by internal $\mathrm{S} / \mathrm{H}$ circuit) only at the moments of synchronization (quantization), and blocks of continuous time - at each step.

Hybrid models allow multi-frequency quantization. To develop them, it is enough to set the periods not by default, but more in a multiple number of times (simulation step). But the quantization period $d T$ should be at least 2-6 times less than the smallest time constant of discrete blocks. When its magnitude is too large, errors introduce at logarithmic phasefrequency characteristic, and not at logarithmic amplitude-frequency characteristic.

\section{Methods}

The model for managing the grain drying process is developed in the VisSim-32 application package. The structure of the mathematical model is block-modular (Figure 1).

Basic data:

- wet grain weight;

- moisture content;

- initial grain temperature;

- the final (boundary) temperature of the grain, above which the grain should not be heated;

- magnetron power.

These parameters can be changed within reasonable limits [2].

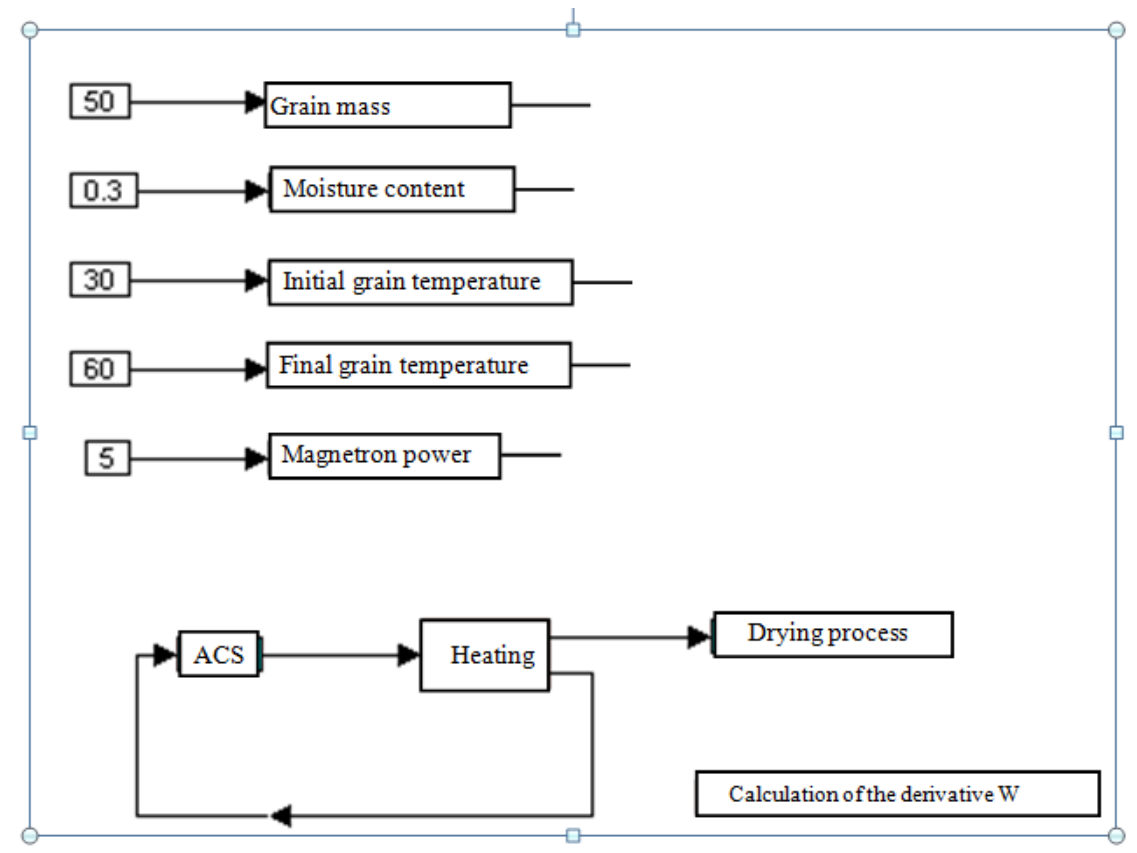

Fig. 1. The structure of the mathematical model.

The block of automatic control system (Figure 2) in the expanded form, simulates a temperature control system in a substance. The grain is heated to the maximum temperature in accordance with the specified technological mode. The converted signal is set to the comparison device from the temperature sensor. When the maximum temperature is reached in a predetermined temperature range, periodic magnetron on-off occurs. Thus, the temperature control mode is relay. 


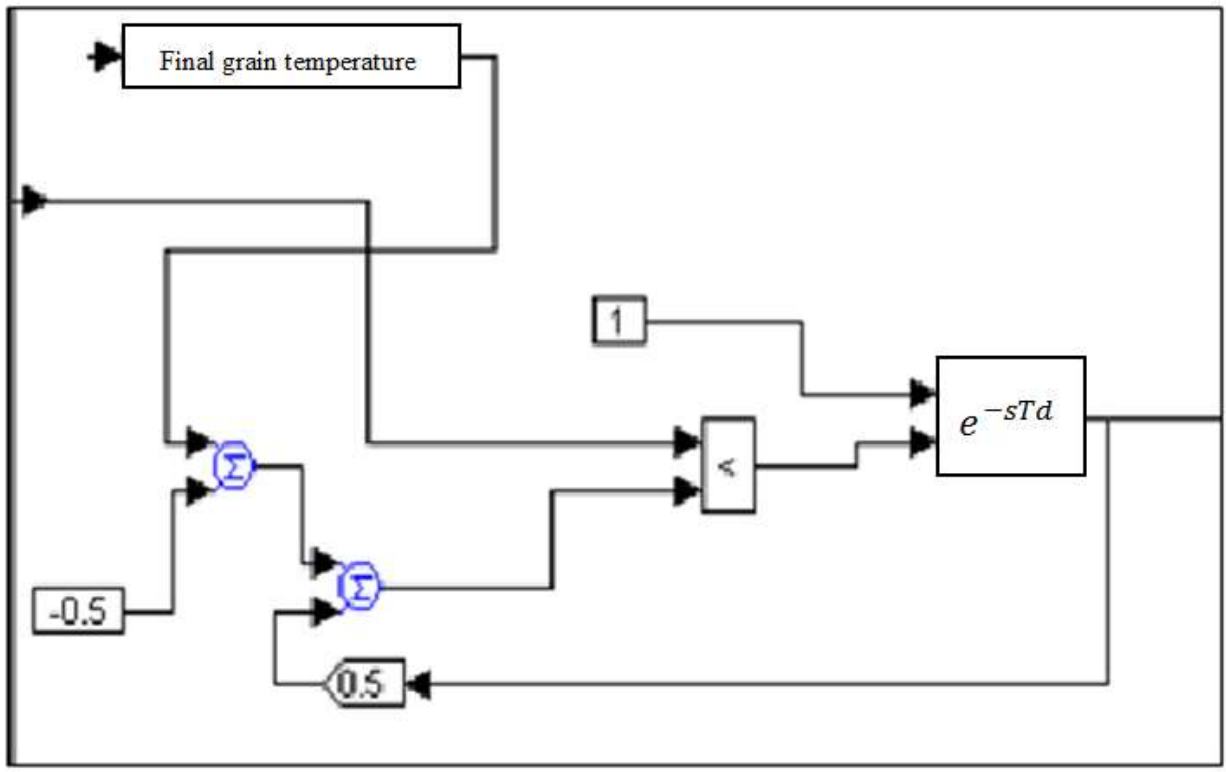

Fig. 2. Temperature control block.

In the "Heating" block, heat exchange processes are modeled with regard to the moisture removal (Figure 3):

a) the amount of heat $\Delta Q_{1}=C_{1} m_{1}\left(T_{f}-T_{0}\right)$ is obtained by the dry part of the grain when heating from the initial temperature $T_{0}$ to the final $T_{f}$. $C_{1}$ is the specific heat content of the dry part of the grain; $m_{1}$ is the mass of the dry part of the grain. The value $m_{1}$ is determined from the expression $m_{1}=m\left(1-W_{0}\right)$, where $m$ is the mass of wet grain, $W_{0}$ is the initial moisture content - basic data;

b) in the system, heat losses through the resonator enclosure due to the temperature difference in the object and the environment $T_{0}$ :

$$
\Delta Q_{2}=k\left(T-T_{0}\right) \Delta \tau ; T>T_{0} ; k=0,02 \frac{\mathrm{kW}}{\operatorname{grad}}
$$

c) the amount of heat is losses continuously due to convective heat exchange with the air flow generated by the operating fans. The average power loss is approximately $0.2 \mathrm{~kW}$. 


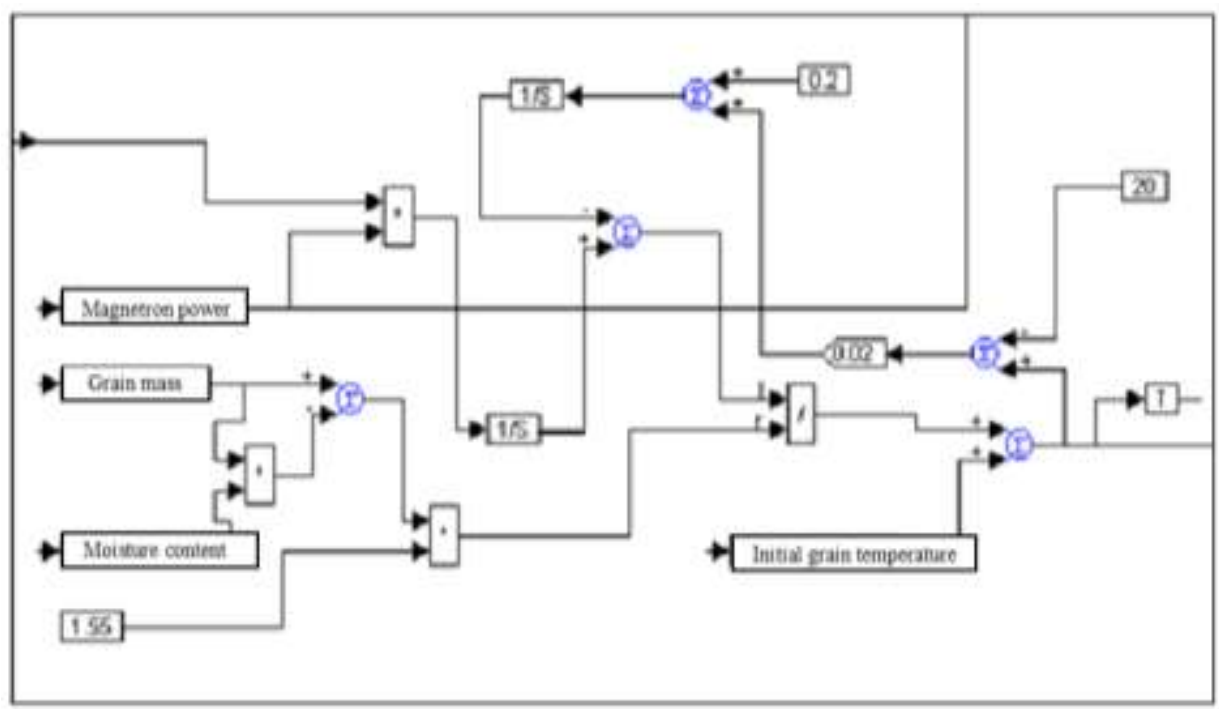

Fig. 3. The block "Heating" in the exposed form.

Since the tangent of the dielectric loss angle of moisture is much larger than the tangent of the loss angle of the dry part of the grain, the temperature of the moisture in an alternating microwave field rises almost instantly with its continuous removal, i.e. the drying process begins immediately after the magnetron is on. The model of this process is represented by the block "Drying Process" (Figure 4).

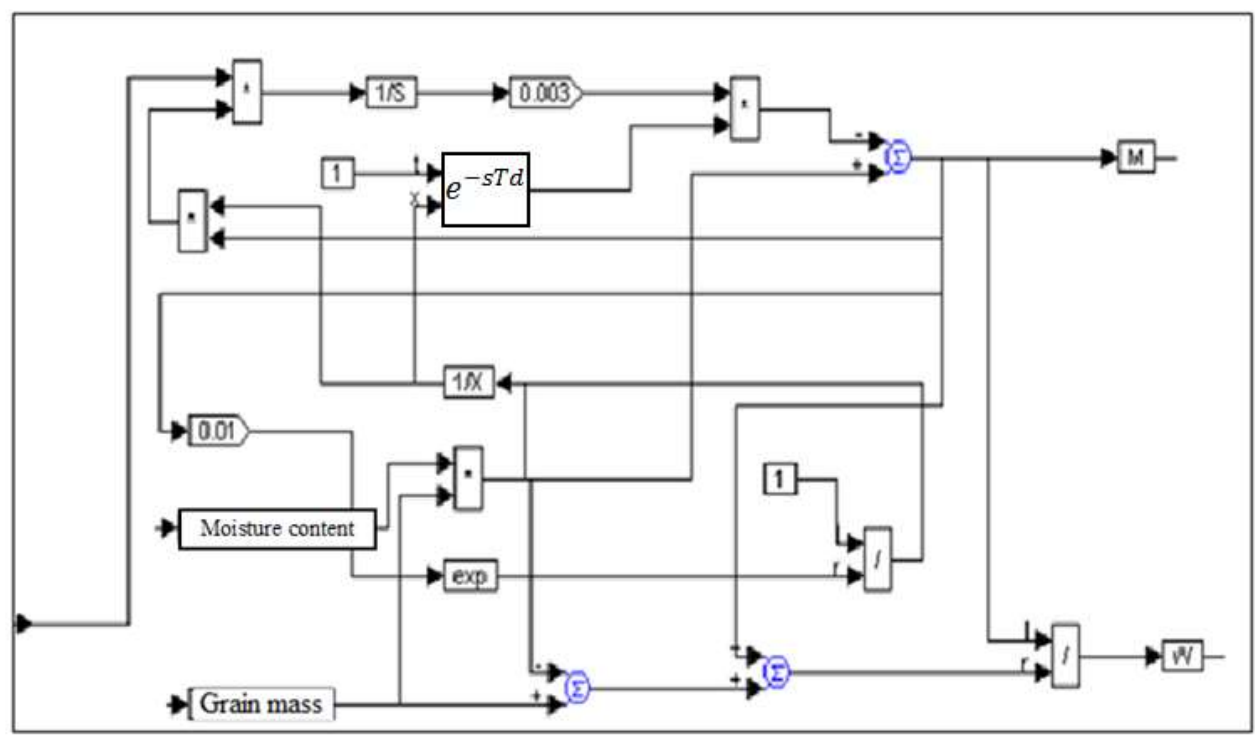

Fig. 4. The Block "Grain Drying Process". 


\section{Results}

From obtained experimental curves $W=f(\tau)$, where $W$ is the relative grain moisture, $\tau$ is the time, it follows that the dependence of moisture on time during the drying process is exponential. In this case, the kinetic equation of drying is

$$
-\frac{d W}{d \tau}=K\left(W-W_{e}\right)
$$

Where $K$ is the kinetic coefficient; $W_{e}$ is an equilibrium moisture content of the material.

Figure 5 shows the graphs of the dependences of the grain temperature and relative moisture content $W$ on the time during the drying. With an initial grain moisture content of $30 \%$, a grain mass of $50 \mathrm{~kg}$, a magnetron power of $5 \mathrm{~kW}$, the drying to a moisture level of $12 \%$ lasts about 20 minutes. With less initial moisture content, the time of drying reduces (Figure 6).

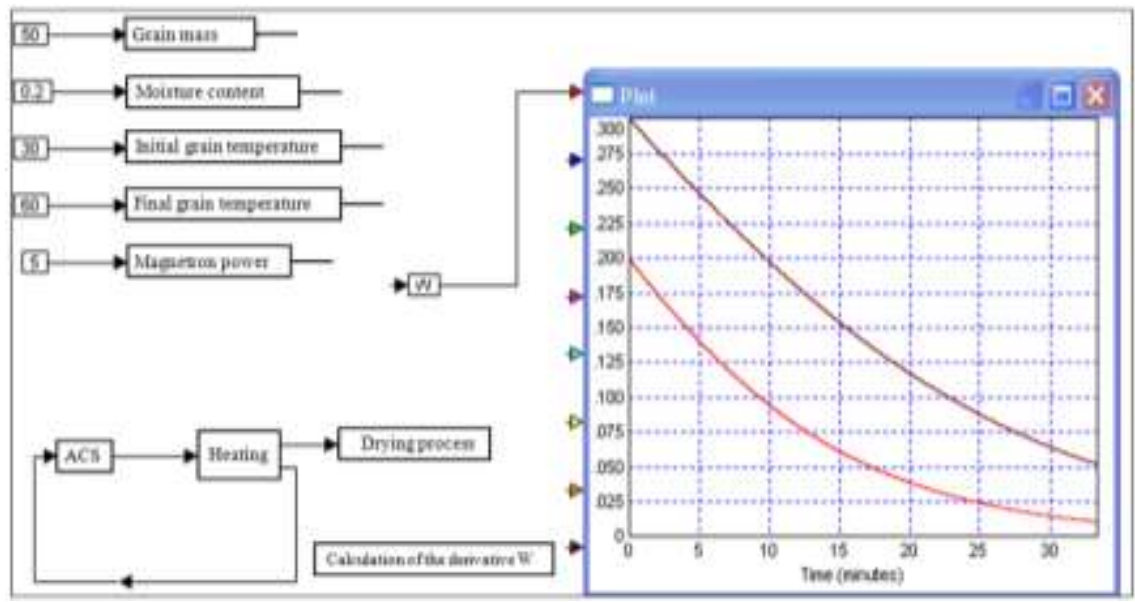

Fig. 5. The drying process at different values of the initial moisture content of the grain.

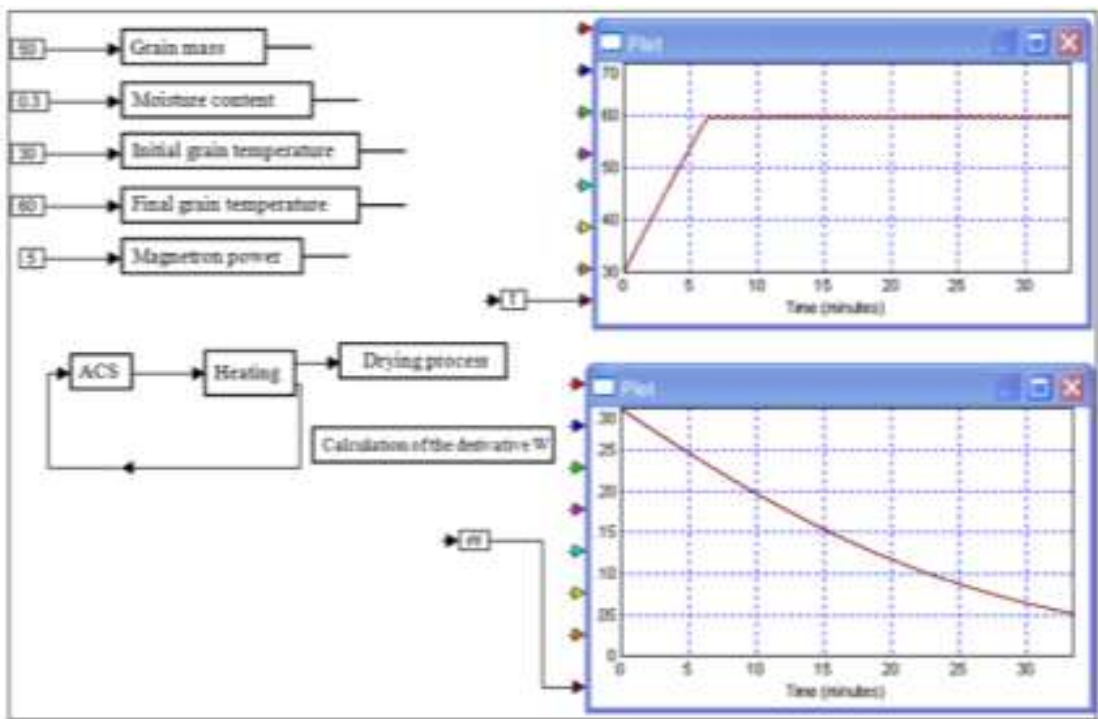

Fig. 6. The dependence of temperature and moisture content on time. 
The block "Calculation of the Derivative $W$ " with the help of a delay link organizes the calculation of the derivative of moisture content with time, i.e. $\frac{d W}{d \tau}$ is a drying rate. The block "Calculation of the Derivative $W$ " in the exposed form is shown in Figure 7.

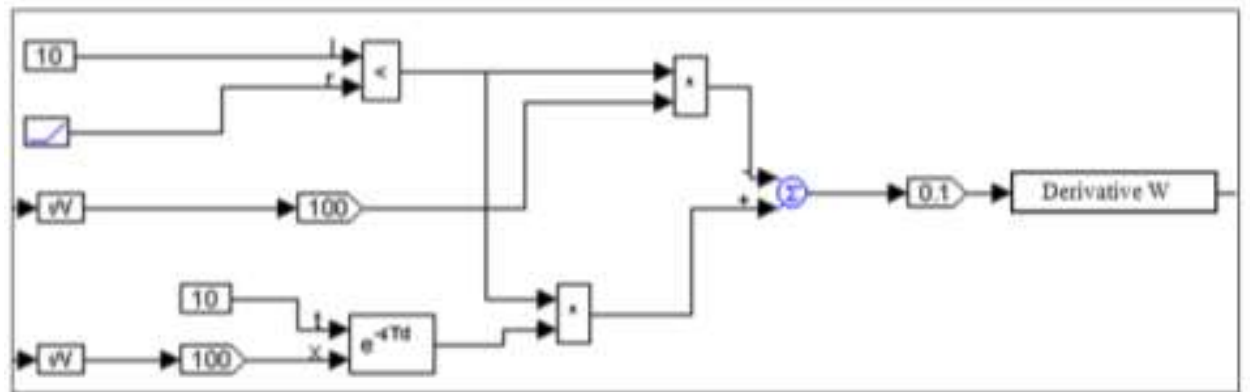

Fig. 7. The block "Calculation of the Derivative $W$ " in the expose form.

Figure 8 shows a graph of the drying rate versus moisture content. It follows from the graph that the drying rate decreases with a decrease in moisture content.

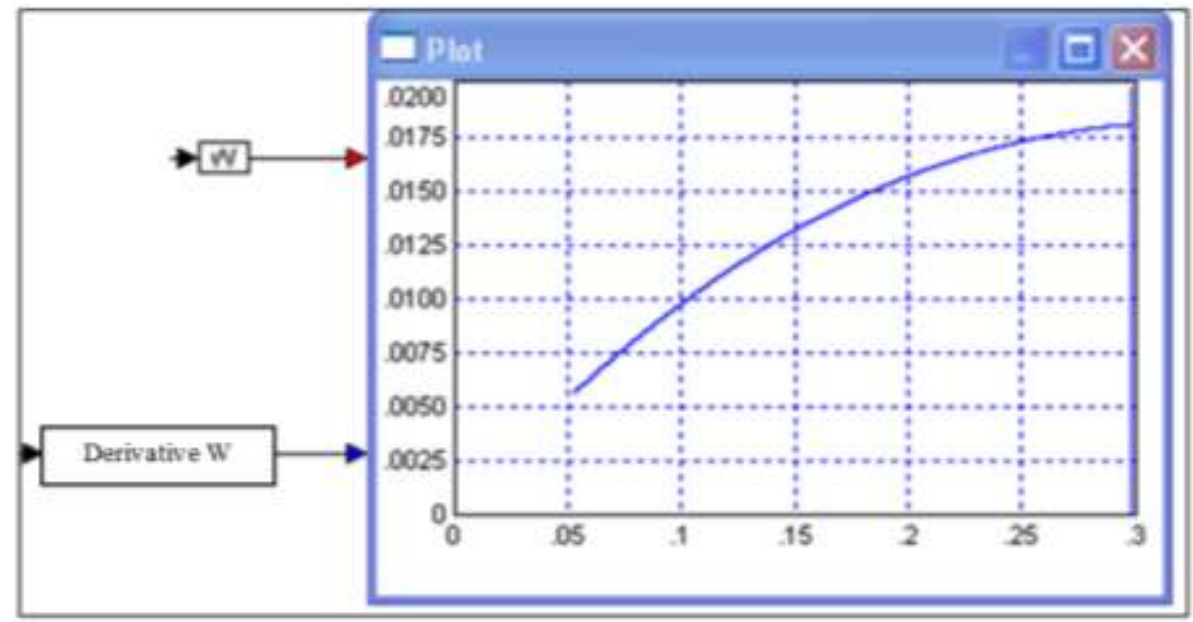

Fig. 8. The dependence of $t$ the drying rate on moisture content.

\section{Conclusion}

The above modeling of the grain drying process makes it possible to assess the current technological process, as well as to explore the potential for optimizing real or introducing new technological processes.

The considered model describes the real process and can be easily changed in the modeling process $[3,4]$.

Optimization of the model can be carried out by multiple run on a PC with a consistent change in its parameters. In the process of the model, one can observe the processes at various stages of drying and, if necessary, change the corresponding parameters, achieving the best results [5].

The mathematical model can be very useful during the unit operation. 


\section{References}

1. K.A. Kuzmin, S.M. Morozov, Inzhenernyy vestnik Dona 2, 60-69 (2018)

2. K.A. Kuzmin, S.M. Morozov, Inzhenernyy vestnik Dona 3, 75-83 (2018)

3. S. Morozov, K. Kuzmin, G. Makarov, MATEC Web Conference 193, 102-115 (2018)

4. S.M. Morozov, V.V. Efimov, K.A. Kuzmin, E.V. Balmashnova, L.I. Kochetkova, Bulletin of Construction Equipment 9, 37-49 (2018)

5. S.M. Morozov, V.V. Efimov, K.A. Kuzmin, E.V. Balmashnova, L.I. Kochetkova, Bulletin of Construction Equipment 9, 62-70 (2018) 